\title{
Variable and polarized emission from $\operatorname{SgrA} *_{\dagger}$
}

\author{
A. Eckart,${ }^{1}$ R. Schödel, ${ }^{1}$ L. Meyer, ${ }^{1}$ C. Straubmeier, ${ }^{1}$ M. Dovčiak, ${ }^{2}$ \\ V. Karas, ${ }^{2}$ M. R. Morris ${ }^{3}$ and F. K. Baganoff ${ }^{4}$ \\ ${ }^{1}$ I. Physikalisches Institut, University of Cologne, Zülpicher Str. 77; D-50937 Köln, Germany \\ ${ }^{2}$ Astronomical Institute, Academy of Sciences, Boční II, CZ-14131 Prague, Czech Republic \\ ${ }^{3}$ Department of Physics \& Astronomy, UCLA, Los Angeles, CA 90095-1547, USA \\ ${ }^{4}$ Kavli Institute for Astrophysics and Space Research, MIT, Cambridge, MA 02139-4307, USA
}

\begin{abstract}
The super-massive black hole in the Galactic Center (Sagittarius A*) is one of the most exciting targets in the sky. At a distance of $\sim 8 \mathrm{kpc}$ it is about one hundred times closer than the second nearest nucleus of a similar galaxy, M31, and therefore the closest galactic nucleus that we can study. Here we report on the modeling of polarized near-infrared flare emission from SgrA* using a model in which a hot spot is moving on a relativistic orbit around the massive black hole. We also summarize the results from simultaneous radio/near-infrared/X-ray measurements of flare emission.
\end{abstract}

Keywords. Galaxy: center - Galaxy: nucleus - accretion, accretion disks - black hole physics radiation mechanisms: non-thermal - relativity

\section{Introduction}

Compelling evidence for a massive black hole at the position of Sagittarius $\mathrm{A}^{*}\left(\mathrm{SgrA}^{*}\right)$ is provided by observations of stellar dynamics and of the variable emission from that position, both in the X-ray and in near-infrared (NIR) domains (see also Eckart, Schödel \& Straubmeier 2005 and references therein). NACO adaptive optics (AO) instrument at the ESO VLT provided the infrared data of the first simultaneous NIR/X-ray flare detections. Repeated measurements have shown that to within less than 10 minutes the brighter X-ray flare events occur simultaneously with the corresponding NIR flares.

Recent near-infrared polarimetric observations with NACO at the VLT UT4 (Yepun) have revealed that some of the 1-2 hour flares exhibit a surprising fine structure in the form of polarized sub-flares that have duration of only about 7-10 minutes and are spaced by about $20 \pm 3$ minutes from peak to peak. These features can successfully be interpreted as emission from hot spots that are on relativistic orbits around the central black hole.

\section{Polarized sub-flares from SgrA*}

We have obtained new polarization data of the variable NIR emission of $\mathrm{SgrA}^{*}$ using the NACO instrument in 2004 and 2005 (Eckart et al. 2006a,b). These new data reveal that some of the typically 60-100 minute long infrared flares are modulated by highly polarized sub-flares with durations of only about 10 minutes (Eckart et al. 2005) with an overall degree of polarization of the order of 10-20\% (Figure 1). In 2005 the main underlying flare was long enough to record three consecutive sub-flares that are consistent with quasi-periodicity of $20 \pm 3$ minutes, similar to the value of $17 \pm 2$ minutes found in previous NACO observations (Genzel et al. 2003; see also Gillessen et al. 2005). This

$\dagger$ Based on observations with CHANDRA and ESO VLT observations 271.B-5019, 073.B-0249, 75.B-0093, 075.B-0113, 076.B-0863, and 077.B-0028. 

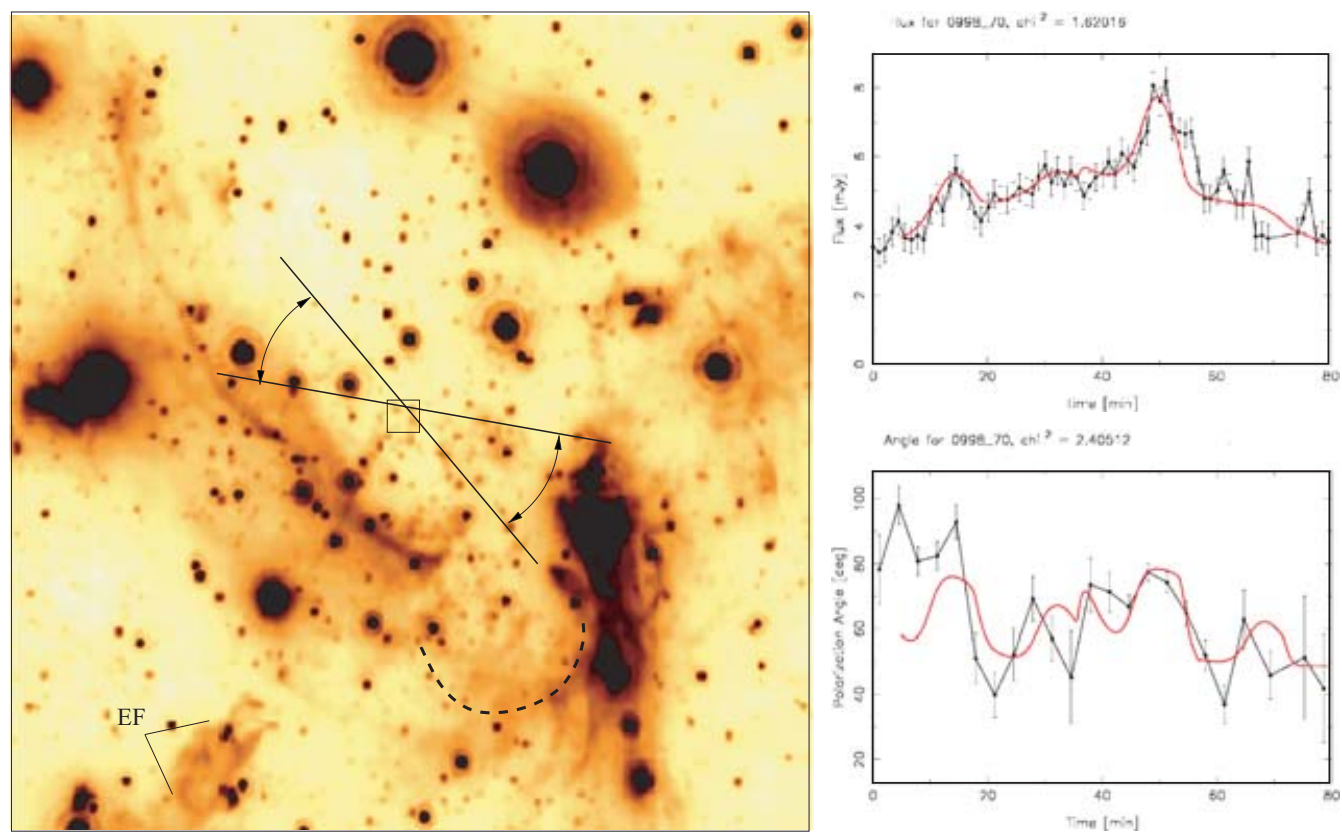

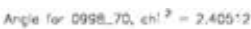

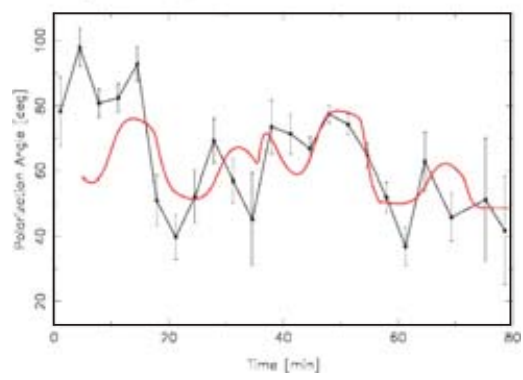

Figure 1. Left: An image of the central $0.5 \times 0.5$ square parsec $(1 \operatorname{arcsec} \sim 0.039 \mathrm{pc})$ of the Galactic Center at a wavelength of $3.8 \mu \mathrm{m}$ using the NACO adaptive optics system at the VLT, showing stars and dust emission from the mini-spiral. The two lines centered on the position of $\mathrm{SgrA}^{*}$ cover the range over which the polarization angle observed in July 2005 and June 2004 varies on the sky. By dashed line we show the approximate location and shape of the mini-cavity. The source EF is described in Eckart et al. (2006b). Right: Total flux (top) and polarization angle (bottom) shown for a flare observed in 2004 (Eckart et al. 2005; Meyer et al. 2006a). In red we show a fit assuming a disk/spot relativistic model (see text for details).

resembles periodicity that has been reported recently for a bright X-ray flare (Bélanger et al. 2006).

The rapid variation of polarized emission is though to be indicative of synchrotron radiation by relativistic electrons and the intrinsic polarization of the sub-flares could be up to $60 \%$. Polarization measurements have the potential to reveal strong-gravity effects of cosmic black holes at significantly better precision than what has been possible until now. By analyzing time-variable polarization degree and the polarization angle, we will be able to set tight constraints on the $\mathrm{SgrA}$ * black hole properties and relativistic models describing the motion and radiation of matter at distances of only a few gravitational radii from the Galactic Center.

Observed polarimetric properties are influenced by the gravitational field of the black hole as light passes near its horizon. Photons follow null geodesics and the Stokes vector is determined by the law of parallel propagation (Connors \& Stark 1977). Therefore, unlike in Newtonian gravity, the polarization angle changes its direction if gravitational field is present and this relativistic phenomenon becomes strong near the black hole. Purely gravitational effects mix up with the intrinsic microphysical properties of gas emitting the light, hence, both gravitational physics and radiation mechanisms must be taken into account when interpreting the observations.

Our preferred model to explain the quasi-periodic polarized flux density fluctuations is that of an under-luminous transient disk, part of which is a spot or a blob orbiting the central black hole (see Figs. 2-3; more details of the relativistic calculations were given 

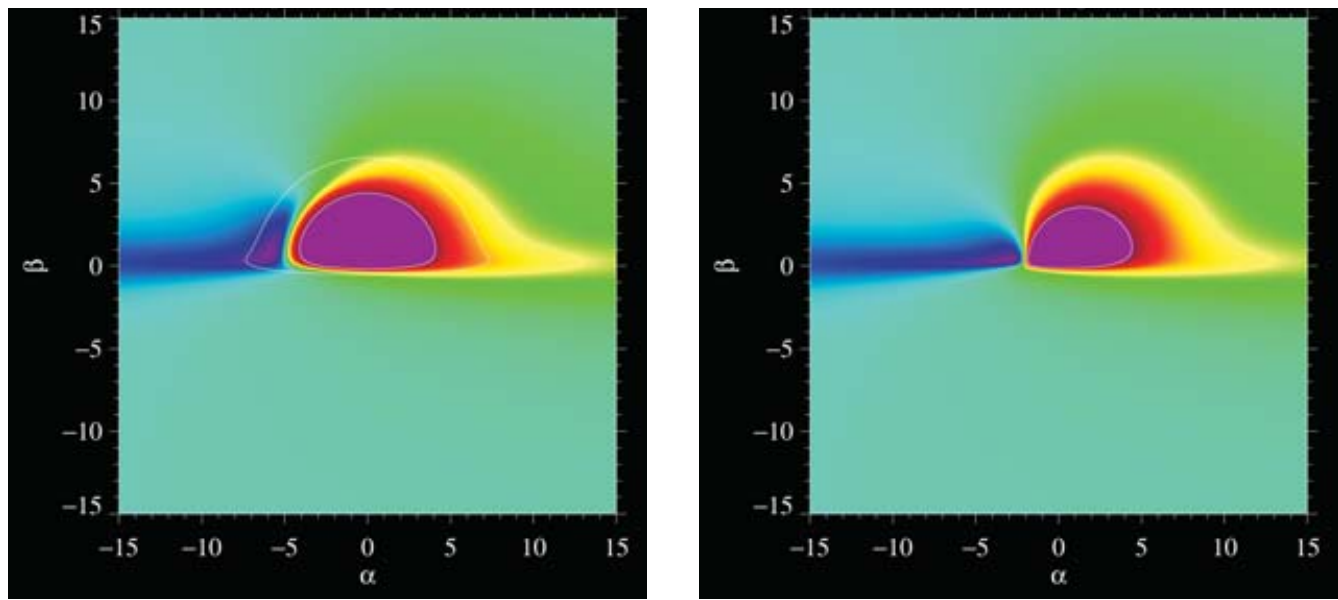

Figure 2. Color-coded levels of the energy shift for a source of light in Keplerian motion around a black hole, as projected onto observer's image plane $(\alpha, \beta)$ at inclination of $i=85^{\circ}$ with respect to the common rotation axis. Left: $a=0$ (non-rotating black hole). Right: $a=1$ (maximally rotating Kerr black hole). Projections of the the black hole horizon and the marginally stable orbit are also indicated.
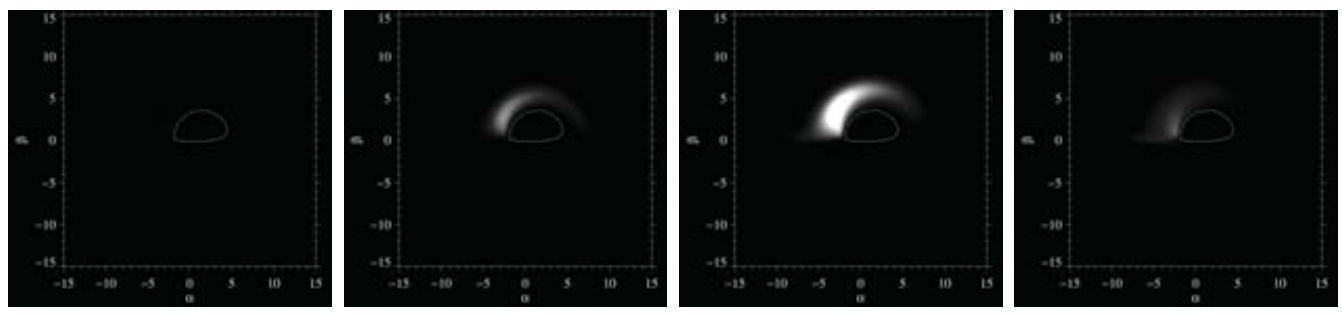

Figure 3. Photometric images of an orbiting hot-spot as seen by an observer at four different orbital phases. The time series covered by the images were calculated for a case of inclination $i=85^{\circ}, a=1$ for a spot at a distance of $4 R_{g}$ (four gravitational radii from the SgrA* black hole; Dovčiak et al. 2004). Models like this can successfully fit the data presented in Eckart et al. (2005, 2006a,b) and Meyer et al. (2006a,b).

in Dovčiak, Karas \& Yaqoob 2004). In this model the temporal variations are explained by relativistic enhancement and subsequent dilution of the radiation flux when the spot is approaching/receding with respect to the observer. At the same time the formation of partial Einstein rings due to gravitational lensing affects the overall polarization of the hot spot.

All these effects are a function of the spot properties as well as the spin parameter of the black hole and the spin orientation with respect to the spot orbit. Another model parameter is the orientation of the magnetic field. A toroidal form of the $B$-field will result in apparent rotation of $E$-field vector. As an alternative, the $B$-field arrangement of the spot may be such that the apparent $E$-field is perpendicular to the disk (see Fig. 4). We found that the minimum spin parameter of $a \sim 0.5$ is consistent with the observed quasi-periodicity. We also found a tendency for high inclinations, and spot radii larger than the last stable orbit. Detailed calculations are presented by Meyer et al. (this volume, and refs. 2006a,b) and Eckart et al.(2005, 2006a).

As an unexpected surprise, for all the bright polarized flares observed between 2003 and 2006 the position angle of the mean $E$-field vector maintains a value of about $60 \pm 30$ degrees on the sky during sub-flares (Meyer et al. 2006a,b). This suggests a preferred 

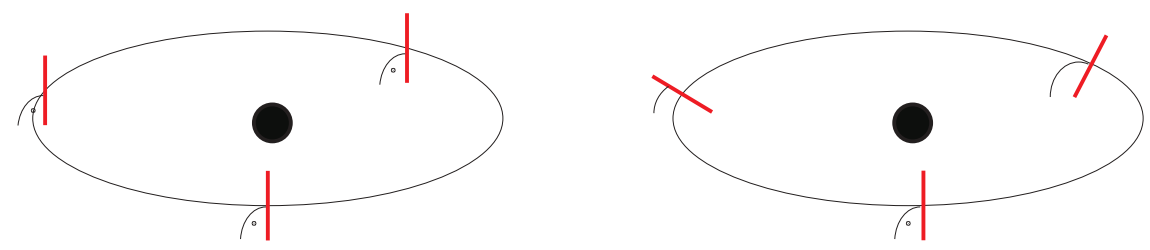

Figure 4. A sketch of two extreme arrangements of the magnetic fields that have been employed to model the hypothetical disk around SgrA*. The $E$-vector (red) for the two magnetic field configurations is shown in a sketch. The massive black hole is represented by the black circle; the ellipse indicates a projected orbit of a hot spot in the equatorial plane. Left: the case where the $E$-vector is constant and perpendicular to the disk. Right: the case of an azimuthal magnetic field; the $E$-vector of the emitted (synchrotron) radiation rotates.

orientation of the overall black hole/disk arrangement with respect to the observer. Future simultaneous observations covering the near-infrared, radio millimeter and sub-millimeter domains will provide a clear discrimination against explanations involving jets. Near the last stable orbit, the expected signatures of a short jet with a length of only a few Schwarzschild radii (the extent of the jet base, i.e., a nozzle) emerging from a disk would likely look almost indistinguishable from signal from a pure disk with orbiting spots.

\section{Simultaneous observations of flares in the NIR and X-ray domains}

Following the first successful experiment coordinated between the VLT and the Chandra satellite during which simultaneous NIR and X-ray flare emission was detected (Eckart et al. 2004; see also Yusef-Zadeh et al. 2006a), new simultaneous NIR/submillimeter/X-ray observations of the SgrA* counterpart were recently presented by Eckart et al. (2006b). In addition to NACO, the Chandra X-ray Observatory as well as the Submillimeter Array on Mauna Kea (Hawaii) and the Very Large Array in New Mexico were involved.

For a total of 4 near-IR flares we found an upper limit for a time lag between the X-ray and NIR flare of $\leqslant 10$ minutes. The NIR/X-ray flares from $\operatorname{SgrA}^{*}$ can be explained with a synchrotron self-Compton (SSC) model involving up-scattered sub-millimeter photons from a compact source component. Inverse Compton scattering of the THz-peaked flare spectrum by the relativistic electrons then accounts for the X-ray emission. This model is in full agreement with the relativistic orbiting spot model described above. The excess flux densities detected in the radio and sub-millimeter may be linked with the NIR flare activity via cooling through adiabatic expansion of a synchrotron component (see also Marrone et al. 2006; Mauerhan et al. 2005). A similar behaviour was recently found in dual wavelength radio data by Yusef-Zadeh et al. (2006b).

Until now only a few hours of overlapping observations were gained between the NIR/X-ray data and the VLA and SMA data; extensive simultaneous data have not been achieved available so far. Future progress will depend on further successful simultaneous observing campaigns. Polarization data from the NIR to the radio as well as (sub-)mm-VLBI and NIR interferometric experiments are also highly desirable to study the details of the accretion process in SgrA*.

\section{Acknowledgements}

This work was supported in part by the Deutsche Forschungsgemeinschaft (DFG) via grant SFB 494. We are grateful to all members of the NAOS/CONICA and the ESO PARANAL team. The X-ray work was supported by NASA through Chandra award 
G05-6093X. VK and MD acknowledge continued support from the Academy of Sciences (ref. 300030510) and the Czech Science Foundation (ref. 205/07/0052).

\section{References}

Bélanger, G., Terrier, R., De Jager, O., Goldwurm, A. \& Melia, F. 2006, Journal of Physics: Conference Series, 54, 420 (astro-ph/0604337)

Connors, P. A. \& Stark, R. F. 1977, Nature, 269, 128

Dovčiak, M., Karas, V. \& Yaqoob, T. 2004, ApJS, 153, 205

Eckart, A., Baganoff, F. K., Morris, M., Bautz, M. W. et al. 2004, A\&A, 427, 1

Eckart, A., Schödel, R. \& Straubmeier, C. 2005, The Black Hole at the Center of the Milky Way (London: Imperial College Press)

Eckart, A., Schödel, R., Meyer, L., Trippe, S., Ott, T. \& Genzel, G. 2006a, A\&A, 455, 1

Eckart, A. Baganoff, F. K., Schäel, R., Morris, M. et al., 2006b, A\&A, 450, 535

Genzel, R., Schd̈el, R., Ott, T., Eckart, A., Alexander, T., Lacombe, F., Rouan, D. \& Aschenbach, B. 2003, Nature, 425, 934

Gillessen, S., Davies, R., Kissler-Patig, M., Lehnert, M. et al. 2005, ESO Messenger, 120, 26

Marrone, D. P., Moran, J. M., Zhao, J.-H. \& Rao, R. 2006, ApJ, 640, 308

Mauerhan, J. C., Morris, M., Walter, F. \& Baganoff, F. K. 2005, ApJ, 623, L25

Meyer, L., Eckart, A., Schödel, R., Duschl, W. J., Muzic, K., Dovčiak, M. \& Karas, V. 2006a, A\&A, 460, 15

Meyer, L., Schödel, R., Eckart, A., Karas, V., Dovčiak, M. \& Duschl, W. J. 2006b, A\&A, 458, L25

Yusef-Zadeh, F., Bushouse, H., Dowell, C. D., Wardle, M., Roberts, D. et al. 2006a, ApJ, 644, 198

Yusef-Zadeh, F., Roberts, D., Wardle, M., Heinke, C. O., Bower, G. C. et al. 2006b, ApJ, 650, 189

Alexander Zakharov: What would be the appropriate size of the emitting region which produces the observed signal in your model?

ANDREAS ECKART: The actual size of the emitting region should be between $\sim 20$ $30 \mu$ arcsec. The spot would then be only a fraction of that size.

Felix Mirabel: Do you favor the model of a rotating hotspot rather than an ejected blob and, if this is the case, for what reason?

AnDREAS ECKART: Quasi-periodicity is a slight argument in favor of the orbiting spot model. On the other hand a short jet has not been excluded and indeed we also consider such possibility in our work. The question whether there is a jet has not yet been solved.

Felix Mirabel: My question was motivated by the fact that we find some kind of similar quasi-periodicity also in GRS1915+105. And if we are dealing with an ejected blob than the mass scalling of timescales does not apply - although at the beginning we also considered a hotspot model, such interpretation was then excluded by imaging.

ANDREAS ECKART: As I mentioned above we do not exclude the possibility of the ejected matter in SgrA* and actually we discuss more details on this idea in our A\&A paper published recently. 


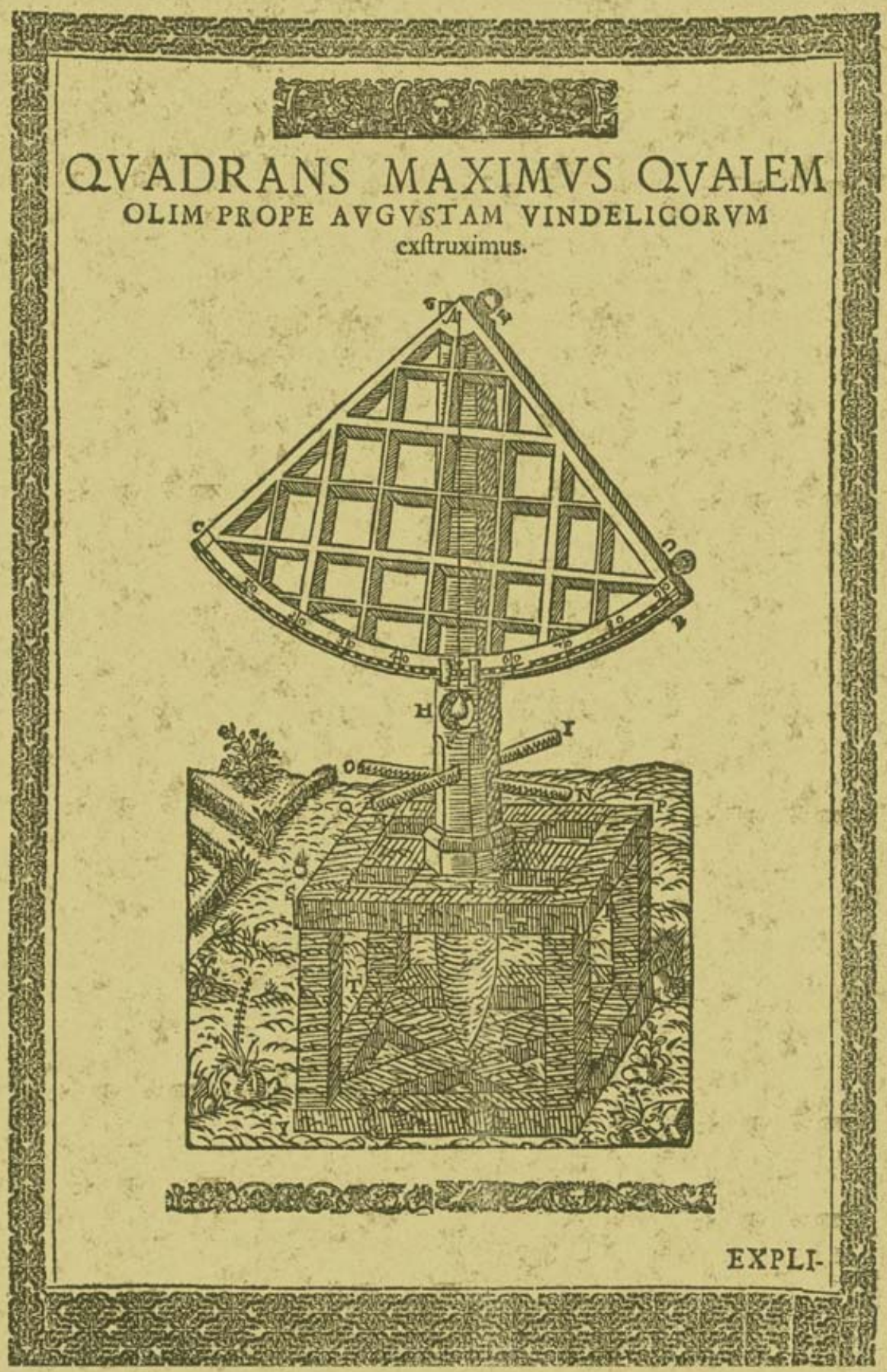

